\title{
CONSIDERAÇÕES ACERCA DA ATUAÇÃO DO ENFERMEIRO NAAPLICABILIDADE DAS DIRETIVAS ANTECIPADAS DE VONTADE
}

Silvana Bastos Cogo', Valéria Lerch Lunardi², Elisabeta Albertina Nietsche ${ }^{3}$

Objetivo: compreender como os enfermeiros entendem a aplicabilidade das diretivas antecipadas de vontade no cuidado em final de vida. Metodologia: pesquisa qualitativa, descritiva e exploratória, realizada com oito enfermeiros de um hospital universitário do Sul do Brasil, em dezembro de 2014, a partir de entrevistas semiestruturadas, submetidas à análise textual discursiva. Resultados: emergiram três categorias: conflitos na assistência ao doente em fase final; vantagens e dificuldades à aplicabilidade das diretivas antecipadas de vontade; benefícios na aplicabilidade das diretivas antecipadas de vontade pela enfermagem. Conclusão: as diretivas antecipadas estão relacionadas ao respeito da autonomia pessoal, entretanto uma prática cercada por medos e receios ao seu cumprimento.

Descritores: Ética em Enfermagem, Diretivas Antecipadas, Autonomia Pessoal.

\section{CONSIDERATIONS REGARDING THE NURSE'S ACTIVITIES IN THE APPLICABILITY OF THE ANTECIPATED INTENTION DIRECTIVE}

Objective: to understand how nurses understand the applicability of early intension directives in the care of the last phases of life. Methodology: qualitative, descriptive and exploratory research, carried out with eight nurses from a university hospital in the south of Brazil, in December 2014, based on semi-structured interviews, submitted to discursive textual analysis. Results: three categories emerged: conflicts in patient care in the final phase; Advantages and difficulties in the applicability of antecipated directives of intention; Benefits in the applicability of the antecipated intension directives by the nursing. Conclusion: The antecipated directives are related to the respect of personal autonomy, however a practice surrounded by fears and concern of its fulfillment.

Descriptors: Nursing Ethics, Antecipated Directives, Personal Autonomy.

\section{CONSIDERACIONES ACERCA DE LA ATUACIÓN DEL ENFERMERO EN LA APLICABILIDAD DE LAS DIRECTIVAS ANTICIPADAS DE VOLUNTAD}

Objetivo: comprender cómo las enfermeras entienden la aplicabilidad de las directrices anticipadas de voluntad en la atención al final de la vida. Metodología: investigación cualitativa, descriptiva y exploratoria, realizada con ocho enfermeras de un hospital universitario en el sur de Brasil, en diciembre de 2014, a partir de entrevistas semiestructuradas sometidos a análisis textual discursiva. Resultados: Tres categorías: surgieron conflictos en la atención prestada a los pacientes en la etapa final; ventajas y dificultades de la aplicación de las directivas anticipadas de voluntad; Beneficia a la aplicabilidad de las directrices anticipadas de voluntad por parte de enfermería. Conclusión: Las instrucciones previas están relacionados con el respeto de la autonomía personal, sin embargo un lugar cercado por los temores y preocupaciones a su cumplimiento.

Descriptores: Ética en Enfermería, Directivas Anticipadas, Autonomía Personal.

1Enferneira. Doutora..Universidade Federal de Santa Maria. Email: silvanabastoscogo@gmail.com

2Enfermeira.Doutora. Universidade Federal do Rio Grande.

${ }^{3}$ Enfermeira. Doutora. Universidade Federal de Santa Maria. 


\section{INTRODUÇÃO}

Os recursos tecnológicos avançam com desmedida velocidade e, na área da saúde, os tratamentos capazes de curar as diversas enfermidades elevaram a expectativa de vida e o prolongamento do tempo de vida saudável das pessoas. Entretanto, diante da possibilidade do uso de avançados recursos tecnológicos, há o risco da perda do bom senso perante um doente em final de vida, prolongando, assim, seu processo de morrer ${ }^{(1)}$

É nessa perspectiva que surgem as Diretivas Antecipadas de Vontade (DAV) que, conforme a Resolução no $1995 / 2012$ do Conselho Federal de Medicina, trata-se de um conjunto de desejos, prévio e expressamente manifestado pelo paciente, sobre cuidados e tratamentos que quer, ou não, receber no momento em que estiver incapacitado de expressar, livre e autonomamente, sua vontade ${ }^{(2)}$. As DAV propõem um desafio de reconhecimento da autonomia dos pacientes nos processos decisórios dos tratamentos que repercutem diretamente nas relações entre médico e paciente, médico e família do paciente, e médico com a equipe assistencial ${ }^{(3)}$.

No cenário internacional, onde há legislação sobre a prática das DAV, como por exemplo, Estados Unidos da América, Espanha, Portugal e Argentina, embora a temática ainda suscite dificuldades de aplicação, sob o ponto de vista do cumprimento pelos profissionais e familiares das vontades manifestadas, além dos limites impostos às restrições de tratamento, ela é bem aceita pela população, pacientes e profissionais da saúde ${ }^{(4-6)}$. No cenário brasileiro, as contribuições sobre as DAV, em geral, envolvem análises teóricas de natureza filosófica e jurídica ${ }^{(1,3,7-8)}$, resultados de pesquisas realizadas com seres humanos ${ }^{(9-13)}$, destacando-se que diversas pessoas, mesmo do meio científico, ainda não conhecem esse tema.

Nessa perspectiva, acredita-se que desvelar o entendimento atribuído pelos enfermeiros às DAV auxiliará na assistência aos pacientes em fase final. A enfermagem, por atuar diretamente com o paciente, e por diversos momentos ser por ele solicitada, assim como por seus familiares, deveria ser conhecedora da possibilidade de opção das DAV e, além disso, não podendo ser alheia ao processo de planejamento, concepção e cumprimento das DAV(1).

Assim, parte-se do pressuposto de que os benefícios das diretivas antecipadas não são exclusividades do paciente, que terá sua vontade assegurada, mas também oferecem respaldo aos profissionais da saúde que disporão de uma maior segurança para agir, uma vez que amparados na vontade expressa do próprio paciente. Destaca-se, também, a tão almejada resolução de problemas éticos e morais, perante os quais os profissionais da saúde são submetidos, quando os pacientes perdem sua capacidade autônoma ${ }^{(13)}$.
Desse modo, com a finalidade de contribuir para a reflexão sobre as questões éticas do final de vida, contribuindo com estudos sobre as DAV com evidências científicas na área da prática dos cuidados em saúde, justamente por ainda não se possuir regulamentação legal no Brasil, e pela carência de especificações sobre sua aplicabilidade pelos profissionais enfermeiros, delineou-se como questão de pesquisa: qual o entendimento da aplicação das DAV para os enfermeiros no cuidado em final de vida?

\section{OBJETIVO}

Compreender como os enfermeiros entendem a aplicabilidade das DAV no cuidado em final de vida.

\section{METODOLOGIA}

Trata-se de uma pesquisa descritiva e exploratória com abordagem qualitativa, realizada em um hospital universitário de uma cidade no centro do estado do Rio Grande do Sul (RS), durante o periodo de dezembro de 2014. Participaram oito enfermeiros, sendo sete do sexo feminino e um do sexo masculino, definidos pela saturação dos dados ${ }^{(14)}$. Os critérios de seleção dos participantes adotados foram: enfermeiros atuantes na unidade de clínica médica e no serviço de internação domiciliar por pelo menos seis meses.

A escolha pelos enfermeiros e referidas unidades justificou-se por entender-se que se caracterizam por atender e assistir doentes em fase final. Consequentemente, tais unidades tratam-se de ambientes repletos de vivências de situações conflituosas e de dilemas éticos suscitados pelo processo de terminalidade.

A coleta dos dados iniciou-se após uma aproximação com os profissionais, a fim de conhecê-los e indagar sobre seu interesse em participar da pesquisa. Os participantes do estudo foram esclarecidos sobre os objetivos e a estratégia de coleta de dados. Após a leitura e assinatura do Termo de Consentimento Livre e Esclarecido, as entrevistas foram gravadas digitalmente.

Os dados foram coletados por meio de entrevistas semiestruturadas realizadas em um único encontro no ambiente hospitalar, em salas privativas, enfocando as seguintes questões norteadoras: respeito à autonomia do paciente, conhecimento e vivências profissionais sobre a aplicabilidade das DAV e a atuação diante da presença das DAV. As entrevistas foram transcritas na íntegra e as falas passaram por correções gramaticais. Após a análise textual discursiva ${ }^{(14)}$, emergiram três categorias: conflitos na assistência ao doente terminal; dificuldades à aplicabilidade das diretivas antecipadas de vontade; e benefícios na aplicabilidade das diretivas antecipadas de vontade pela enfermagem. 
O estudo obedeceu aos preceitos éticos e obteve aprovação do Comitê de Ética em Pesquisa na Área da Saúde da Universidade Federal do Rio Grande, sob o Parecer nㅇ. $168 / 2014$. Os depoimentos foram identificados por código (ENF) seguido de número ordinal, de forma a garantir o sigilo e o anonimato dos participantes.

\section{RESULTADOS}

A partir da análise dos dados, emergiram três categorias, que são apresentadas logo a seguir:

\section{Conflitos na assistência ao doente em fase final}

$O$ (des)respeito às vontades dos pacientes em fase final de vida, as incertezas e ambiguidades quanto as condutas de tratamento pela equipe multiprofissional, a fragilidade de interação entre os profissionais da saúde, as incertezas de diagnóstico e prognóstico do paciente e os riscos de punições legais diante da limitação de um esforço terapêutico são questões conflituosas que podem ocasionar desconfortos ao encarar a situação de cuidados ao doente em final de vida, especificamente à possibilidade de aplicabilidade das DAV: Os profissionais ficariam mais tranquilos e seguros em relação às condutas, porque é angustiante. (...) tu vais reanimar só por que a familia está ali (ENF.7); Os riscos de processos contra a enfermagem seriam menores, porque, muitas vezes por deficiências de registros e comunicação, ficamos fragilizados legalmente (ENF.2); Às vezes um plantonista diz uma coisa e outro plantonista outra conduta. O que fazer? (ENF. 5)

\section{Dificuldades à aplicabilidade das diretivas antecipadas de vontade}

A prática das DAV é incipiente no Brasil e suscita questionamentos quanto a sua aplicabilidade. À enfermagem, parte integrante da equipe que atua nos cuidados e aplicação das limitações de terapêutica em final de vida, o desafio é aparente considerando as dificuldades que a aplicação das DAV impõe no contexto da assistência de enfermagem.

Nesse âmbito, há limitações na aplicação das DAV pela enfermagem, pela inexistência de uma legislação que subsidie essa conduta repercutindo em uma atuação centrada no medo de punições legais, além da falta de sincronia nas condutas diante de uma solicitação do paciente. Soma-se, ainda, o receio pelo fato de cumprir algo que foi manifestado previamente, mas que, talvez, não corresponda aos desejos no presente; além disso, a possibilidade do enfermeiro abandonar o paciente, considerando a ideia que ele não quer os investimentos para prosseguir com a sua vida: $E u$ faria tudo que está dentro do legal, eu não iria me expor e responder um processo. As pessoas fazem algo não por conviç̧ão, mas para não se incomodar (ENF8); Eu fico com receio e medo; supondo que o paciente faça (DAV) e precise usar, se acontecer e querer mudar a ideia e não poder (ENFI). Me preocupa ele ter pedido que não fosse feito nada e a gente não vai mudar de decúbito, porque ele não quer ser reanimado, intubado. Há um descaso no cuidado e ele pode ser visto com um olhar diferente (ENF4).

\section{Beneficios na aplicabilidade das diretivas antecipadas de vontade pela enfermagem}

Em geral, o conceito ea prática das DAV são desconhecidos pelos profissionais enfermeiros; entretanto, na pesquisa, as DAV se constituíram como a possibilidade de, a partir do cumprimento dos desejos manifestados previamente pelo paciente, respeitar sua automomia pessoal em final de vida, propiciar o respaldo aos enfermeiros que atuarão amparados na vontade expressa do paciente, além de minimizar os conflitos entre os profissionais, bem como dos familiares do doente diante das decisões que envolvem o final de vida: Com as DAV a gente faz a vontade do paciente que estava consciente quando decidiu (ENF.5); Os riscos de processos contra a enfermagem seriam menores, porque, muitas vezes por deficiências de registros e comunicação, ficamos fragilizados legalmente (ENF.2); Eu me sentiria amparada, pois trabalhamos com equipes grandes e isso ajudaria a diminuir o conflito profissional e ajudaria a instituição e as decisões não ficariam só para os familiares (ENF.8).

\section{DISCUSSÃO}

Nos últimos anos, muito se tem observado acerca da inserção dos assuntos relacionados à assistência aos doentes em fase final de vida, especialmente as DAV, o que significa tangenciar as mais delicadas crenças e convicções, uma vez que possibilita que as próprias pessoas realizem escolhas importantes acerca do rumo de suas vidas, ampliando-as ou as abreviando ${ }^{(15)}$. Assim, as DAV desmistificam a cultura centrada no paternalismo, que reduz o indivíduo a um paciente que deve aguardar, resignada e submissamente, que deliberações acerca de sua vida sejam tomadas por outros, sem que ele possa se manifestar ou decidir como quer ser tratado ou que tipo de práticas de intervenção está disposto a $\operatorname{aceitar}^{(7)}$

Nesse sentido, as decisões que envolvem as limitações de esforço terapêutico, além da interação paciente e família, não devem ser adotadas sem que ocorra uma decisão multidisciplinar, necessidade também identificada pelos enfermeiros entrevistados, especialmente, pelo contexto permeado por questionamentos e incertezas, insuficiente interação profissional e temor de punições. Os estudos apontam que as DAV contribuem no sentido de amenizar aflições dos familiares quanto à responsabilidade de decidir sobre as condutas nas questões relacionadas ao fim da 
vida ${ }^{(6,16-17)}$, também, evidenciados pelos enfermeiros, como verbalizado nos depoimentos.

A decisão de cada profissional deve considerar o conhecimento científico que sustenta o seu exercício profissional e a intervenção que vai prestar ${ }^{(18)}$; ou seja, para que as DAV sejam cumpridas, o médico e o enfermeiro decidem as intervenções baseadas, respectivamente, no conhecimento da medicina e no conhecimento científico da enfermagem amparados por lei de regulamentação profissional, o que reduziria as dificuldades evidenciadas pelos enfermeiros. Mesmo que a decisão não seja construída em equipe, sua implementação requer, necessariamente, a intervenção do médico e do enfermeiro, podendo acontecer que um decida em um determinado sentido e outro em sentido inverso ${ }^{(18)}$.

Os enfermeiros reconhecem que desempenhariam ação fundamental no exercício do direito à autodeterminação, facilitando a tomada de decisão dos doentes. São profissionais presentes não apenas no momento da morte, mas em todo o processo em que o doente e seus familiares tomam decisões de fim de vida $^{(19)}$. Os enfermeiros devem respeitar, reconhecer e realizar ações que garantam o direito da pessoa ou de seu representante legal, de tomar decisões sobre sua saúde, tratamento, conforto e bem estar e suas ações necessitam visar a preservação da autonomia das pessoas ${ }^{(20)}$.

A relevância da enfermagem, frente às DAV, deve-se também a um problema frequente, ou seja, a decisão de não realizar determinado procedimento, de não prestar um cuidado ou uma intervenção. Essas são sempre as decisões mais dificeis ${ }^{(18)}$, também percebida nas falas dos enfermeiros, principalmente diante do necessário apoio legal para suas tomadas de decisão, apesar de se constituírem no respeito aos desejos manifestos do paciente. $O$ enfermeiro assume deveres para proteger e salvaguardar os direitos do cidadão a quem presta cuidados. O Código de Ética dos Profissionais de Enfermagem prevê que a enfermagem deve respeitar o pudor, a privacidade e a intimidade do ser humano, em todo seu ciclo vital, inclusive nas situações de morte e pós-morte, sendo proibida de promover a eutanásia ou participar em prática destinada a antecipar a morte do paciente ${ }^{(20)}$

Ao ser confrontado com uma vontade expressa pelo doente, por vezes, o profissional de saúde ou cuidador debate- se com situações complexas e questiona-se sobre a forma como deve agir perante o princípio da autonomia pessoal. É necessário o conhecimento das competências, deveres e obrigações profissionais, de forma a respeitar e proteger a vida como um direito fundamental das pessoas ${ }^{(1)}$. Ademais, em pesquisa realizada no RS com 23 profissionais da enfermagem, embora as DAV sejam reconhecidas como direito do paciente ou de seu familiar, elas podem entrar em conflito quando são diferentes das práticas da instituição, podendo não serem cumpridas; fundamentando-se no sentido de proteger, por não estarem de acordo com as práticas habituais dos serviços de saúde, no intuito de preservar a vida ou de proporcionar conforto ao paciente ${ }^{(10)}$.

Em vista disso, é que, recentemente, em pesquisa realizada no Brasil, foi constatado que as implicações legais são as que mais interferem nas ações dos profissionais da saúde diante das questões de fim de vida, implicando deixarem de realizar o que acreditam ser o melhor para seus pacientes ${ }^{(13)}$, o que também pode ser depreendido nas manifestações de entrevistados. O fato de um enfermeiro respeitar ou não a vontade do doente, depende de uma escolha pessoal. Existem vários fatores, associados à cultura, história pessoal, experiência, valores, dentre outros, que contribuem para uma tomada de decisão, mas que não excluem seu caráter de liberdade porque o enfermeiro está consciente das suas possibilidades, pois possui o conhecimento acerca da sua profissão e da organização do seu trabalho(19).

Em pesquisa realizada com 139 enfermeiros, em Portugal, para conhecer a percepção sobre as DAV, constatou-se que cerca de $95 \%$ dos enfermeiros não têm experiência de situações em que o doente pode decidir apoiando-se em suas DAV, nem vivenciou situações em que o doente tenha sido informado sobre esse direito(1), o que também foi constatado nas entrevistas realizadas. Ainda, $72,7 \%$ dos enfermeiros manifestaram-se disponíveis para refletir com o doente a elaboração do documento; contudo, $45,3 \%$ só o fariam se o doente ou os familiares o solicitassem ${ }^{(1)}$

Os resultados desse estudo devem ser interpretados à luz de várias limitações. Essa pesquisa foi realizada em apenas duas unidades de um hospital na região central do Rio Grande do Sul, cujas experiências de enfermeiros podem 
diferir, em outros locais e ambientes hospitalares. Entretanto, considera-se que esses resultados conferem profundidade ao entendimento que as DAV constituem uma alternativa para proporcionar a autonomia do doente, a partir do cumprimento das suas vontades, apesar das limitações e conflitos que dificultam sua efetivação no contexto hospitalar.

\section{CONCLUSÃO}

A aplicabilidade das DAV está longe de encerrar as polêmicas que permeiam esse assunto, relacionadas à sua aplicação; à expectativa que a vontade das pessoas corresponda à decisão final; à inserção do profissional de saúde no processo das DAV e se esse pode ou tem capacidade para alterar a decisão do paciente, além de outras dúvidas. Contudo, há que se destacar que essa prática está vigente no Brasil e a preocupação com os procedimentos a serem realizados no fim da vida devem ser inseridos nos espaços da sociedade, em especial nos espaços de formação dos profissionais da saúde, para que, efetivamente, seja garantido o direito a autonomia pessoal.

\section{REFERÊNCIAS}

1. Neves MEO. Percepção dos Enfermeiros sobre Diretivas Antecipadas de Vontade [dissertação]. Viseu: Escola Superior de Saúde de Viseu; 2013. 135p.

2. Conselho Federal de Medicina (BR). Resolução nㅇ․ 1.995, de 9 de agosto de 2012. Dispõe sobre as diretivas antecipadas de vontade dos pacientes [Internet]. Diário Oficial da União 31 ago 2012 [atualizado em 28 maio 2015; acesso em 05 set 2013]; Seção 1:269-70. Disponivel em: http://www. portalmedico.org.br/resolucoes/CFM/2012/1995_2012.pdf

3. Alves CA, Fernandes MS, Goldim JR. [Advance directives: a new challenge to the doctor-patient relationship]. Rev HCPA [Internet]. 2012 [updated 2015 May 28; cited 2014 Nov 05];32(3):358-62. Available from: http://seer.ufrgs.br/index.php/hcpa/article/view/33981/22041 Portuguese.

4. Cucalón-Arenal JM, Marin-Ibáñez A, Cia-Gómez P. Blay-Cortés MG. [University students' opinions regarding end-of-life care]. FEM [Internet] 2013 [updated 2015 May 28; cited 2014 Nov 05];16(3):181-6. Available from: http://scielo.isciii.es/pdf/fem/vl6n3/original7.pdf Spanish.

5. Evans N, Pasman HR, Vega Alonso T, Van den Block L, Miccinesi G Van Casteren V, et al. End-of-life decisions: a cross-national study of treatment preference discussions and surrogate decision-maker appointments. Plos One [Internet]. 2013 [updated 2015 May 28; cited 2014 Nov 05];8(3):57965. Available from: http://www.ncbi.nlm.nih.gov/ pubmed/23472122

6. Keam B, Yun YH, Heo DS, Park BW, Cho CH, Kim S, et al. The attitudes of Korean cancer patients, family caregivers, oncologists, and members of the general public toward advance directives. Support Care Cancer [Internet]. 2013 May [updated 2015 May 28; cited 2014 Nov 05];21(5):1437-44. Available from: http://www.ncbi.nlm.nih.gov/pubmed/23262809

7. Bussinguer ECA, Barcellos IA. [The right to live one's own death and its constitutionality]. Cien Saude Colet [Internet]. 2013 [Internet]. 2013 [updated 2015 May 28; cited 2014 Nov 03];18(9):2691-98. Available from: http://www.scielo.br/pdf/csc/v18n9/v18n9a24.pdf Portuguese.

8. Dadalto L. [Legal repercussions of the Resolution CFM 1.995/12]. Rev Bioét [Internet]. 2013 [updated 2015 May 28; cited 2014 Nov 03];21(1):106-

12. Available from: http://revistabioetica.cfm.org.br/index.php/revista_ bioetica/article/view/791/861 Portuguese.

9. Dadalto L, Tupinambás U, Greco DB. [Advanced directive: a brasilian model]. Rev Bioét [Internet]. 2013 [updated 2015 May 28; cited 2014 Nov 05];21(3):463-76. Available from: http://www.scielo.br/pdf/bioet/v2ln3/ allv2ln3.pdf Portuguese.

10. Cassol PB, Quintana AM, Velho MTAC. Diretiva antecipada de vontade percepção de uma equipe de enfermagem da hemato-oncologia. J Nurs Health, 2015:5(1):04-13.
11. Chehuen Neto JA, Ferreira RE, Silva NCS da, Delgado ÁH de A, Tabet CG, Almeida GGet al . Living will: What do healthcare professionals think about it?. Rev. Bioét. [Internet]. 2015 Dec [cited $2016 \mathrm{Apr} 01$ ] 23(3):572-582. Available from: http://www.scielo.br/scielo.php?script=sci_ arttextEpid=S1983- 80422015000300572\&lng=en. http://dx.doi. org/10.1590/1983-80422015233094

12. Silva JAC da, Souza LEA de, Costa JLF, Miranda H da C. Knowledge of medical students regarding living wills. Rev. Bioét. [Internet]. 2015 Dec [cited 2016 Nov 01] ; 23( 3 ): 563-571. Available from: http://www.scielo.br/ scielo.php?script=sci_arttext\&pid=\$1983-80422015000300563\&lng=en http://dx.doi.org/10.1590/1983-80422015233093.

13. Cogo SB, Lunardi VL, Quintana AM, Girardon-Perlini NMO, Silveira RS. Challenges to implementation of advance directives of will in hospital practice. Rev Bras Enferm [Internet]. 2016:69(6):969-76. DOI: http://dx.doi. org/10.1590/0034-7167-2016-0085

14. Moraes R, Galiazzi MC. Análise Textual Discursiva. $2^{a}$ ed. Rev. Ijuí: Unijuí: 2013.

15. Xavier MP. Declaração prévia para o fim da vida: Análise a partir do contexto brasileiro contemporâneo. In: DADALTO, Luciana (coord). Diretivas Antecipadas de Vontade: Ensaios sobre o direito à autoderteminação. 1. ed Belo Horizonte: Letramento, 2013. 281p.

16. Sarmiento-Medina MI, Vargas-Cruz SL, Velásquez-Jiménez CM. Jaramillo MS. [Terminally-ill patients' end of life problems and related decisions]. Rev Salud Public [Internet]. 2012 Jan-Feb [updated 2015 May 28 cited 2014 Nov 03];14(1):116-28. Available from: http://www.scielosp.org/ pdf/rsap/vl4nl/vl4nlalO.pdf Spanish.

17. Schenker Y, Crowley-Matoka M, Dohan D, Tiver GA, Arnold RM, White DB. I don't want to be the one saying 'we should just let him die': intrapersonal tensions experienced by surrogate decision makers in the ICU. J Gen Intern Med [Internet]. 2012 Dec [updated 2015 May 28; cited 2014 Nov 03]:27(12):1657-65. Available from: http://www.ncbi.nlm.nih.gov/ pubmed/23011253

18. Deodato S. Manifestação antecipada de vontade e exercício de Enfermagem. Ordem dos enfermeiros, n. 36, 2010.

19. Przenyczka RA, Lenardt MH, Mazza V de A, Lacerda MR. O paradoxo da liberdade e da autonomia nas ações do enfermeiro. Texto contexto - enferm. [Internet]. 2012 June [cited 2016 Out 20]; 21(2): 427431. Available from: http://www.scielo.br/scielo.php?script=sci_ arttext\&pid=S010407072012000200022\&lng=en. http://dx.doi. org/10.1590/S0104-07072012000200022.

20. Cofen. Resolução 311/2007. Aprova a Reformulação do Código de Ética dos Profissionais de Enfermagem. Disponivel em: http://www.soleis.adv.br/ codigoeticaenfermagem.htm. Acesso em: 10 jun 20 